Questions vives

\section{Questions Vives}

Recherches en éducation

$N^{\circ} 25 \mid 2016$

L'activité des enseignants face au décrochage scolaire : Quelles difficultés et quelles mises en œuvre professionnelles?

\title{
Grangeat, M., Understanding Science Teachers' Professional knowledge Growth
}

Rotterdam : Sense Publisher

\section{Patrice Venturini}

\section{(2) OpenEdition}

1 Journals

\section{Édition électronique}

URL : http://journals.openedition.org/questionsvives/1953

DOI : 10.4000/questionsvives. 1953

ISSN : $1775-433 \mathrm{X}$

Éditeur

Université Aix-Marseille (AMU)

\section{Édition imprimée}

Date de publication : 7 juillet 2016

ISBN : 978-2-912643-49-0

ISSN : $1635-4079$

\section{Référence électronique}

Patrice Venturini, « Grangeat, M., Understanding Science Teachers' Professional knowledge Growth », Questions Vives [En ligne], № 25 | 2016, mis en ligne le 05 septembre 2016, consulté le 23 septembre 2020. URL : http://journals.openedition.org/questionsvives/1953 ; DOI : https://doi.org/10.4000/ questionsvives. 1953

Ce document a été généré automatiquement le 23 septembre 2020.

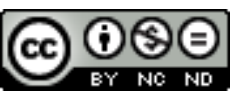

Questions Vives est mis à disposition selon les termes de la licence Creative Commons Attribution Pas d'Utilisation Commerciale - Pas de Modification 4.0 International. 


\title{
Grangeat, M., Understanding Science Teachers' Professional knowledge Growth
}

Rotterdam : Sense Publisher

\author{
Patrice Venturini
}

\section{RÉFÉRENCE}

Grangeat, M. (2015). Understanding Science Teachers' Professional knowledge Growth. Rotterdam: Sense Publisher

1 L'adéquation de la formation professionnelle aux exigences et aux mutations des contextes de travail constitue un enjeu important pour toute société. Elle passe notamment par la formalisation des connaissances professionnelles et la modélisation de leurs processus d'évolution. C'est précisément ce que propose cet ouvrage de 230 pages dans le cas des professeurs de sciences. Ces professionnels ont un rôle vital pour notre société technoscientifique puisque leur fonction est de transmettre à la nouvelle génération la culture scientifique élaborée par les générations précédentes. C'est dire l'importance de toute avancée permettant d'améliorer leur formation, d'autant que "it is what teachers know, do, and care about which is very powerful in the learning equation" (Hattie, 2003). Aussi, la thématique abordée par l'ouvrage constitue-t-elle son premier intérêt, même si elle ne date pas d'aujourd'hui.

2 En effet, Shulman évoquait déjà en 1987 une base de connaissances nécessaire pour enseigner, incluant sept composantes, les connaissances sur le contenu à transmettre, les connaissances pédagogiques générales (Pedagogical Knowledge ou PK), les connaissances sur le curriculum, les connaissances pédagogiques relatives à la transmission d'un contenu particulier (Pedagogical Content Knoweldge ou PCK), les connaissances sur les élèves, sur les contextes éducatifs, ou encore sur les visées éducatives. On lui doit l'introduction des PCK, "that special amalgam of content and 
pedagogy that is uniquely the providence of teachers [...] PCK represents the blending of content and pedagogy into an understanding of how particular topics, problems, or issues are organized, represented, and adapted to diverse interests and abilities of learners, and presented for instruction." (Shulman, 1987, p. 8). Dans une catégorisation ultrieure proposée par Grossman en 1990 (p. 5), les PCK sont supposées avoir l'impact le plus important sur l'action des professeurs en classe. Aussi n'est-il pas surprenant que la recherche en Science Education se soit emparée de ce concept et ait développé de nombreux modèles faisant une part importante aux PCK (voir par exemple Magnusson, Krajcik \& Borko, 1999, p. 99 ou encore Abell, 2007, p. 1107). La diversité était telle qu'il est apparu nécessaire en 2012 de réfléchir à l'unification des modélisations proposées et de baliser en conséquence les nouvelles recherches à mener. C'est l'objectif que s'est fixé le PCK Summit (Biological Sciences Curriculum Study, 2012) qui a permis de produire une nouvelle formalisation, "Teacher Professional Knowledge and Skills including PCK" (Gess-Newsome, 2015) supposée faire consensus en Science Education, au moins provisoirement. A cette occasion, la définition initiale du PCK a été étendue aux connaissances souvent implicites mises en jeu dans l'action, au-delà de la base de connaissances explicites qu'il englobait classiquement. C'est ce modèle qui est utilisé et/ou discuté dans la plupart des douze chapitres de l'ouvrage, rédigé par des chercheurs français et européens et structuré en 3 sections, une introduction et une conclusion.

Outre la présentation des différentes contributions et celle du modèle issu du PCK summit, l'introduction s'attache à dessiner les contours de l'arrière-plan qu'elles partagent. Ainsi, elles conçoivent toutes le développement des connaissances professionnelles comme un processus continu "that depends on the repertoire of actions that are available within the community, on the social context in which teaching is performed, and on the artefacts and resources that exist in the environnement" (p. 2), mettant en jeu des connaissances implicites et explicites liées à la fois à l'expérience personnelle des enseignants et aux artefacts culturels et sociaux à leur disposition. Dans tous les cas, ce développement prend appui soit sur des évènements inattendus survenant dans la classe, soit sur des programmes de formation basés sur la conception itérative de séances et l'analyse de leur déroulement. En formalisant les éléments supposés partagés par l'ensemble des contributeurs, probablement s'agit-il aussi pour le directeur de l'ouvrage de dégager ce qui fait son unité au-delà de sa thématique, un exercice souvent difficile quand les recherches présentées dans chacun des chapitres sont autonomes et indépendantes les unes des autres, même si ici, il faut le souligner, quasiment toutes réfèrent à un seul et même modèle de PCK qui assure une certaine continuité entre elles.

4 La section 1 intitulée "Performing efficient teaching: role of the content to be taught" concerne plus largement les liens entre connaissances du contenu à enseigner (Content knowledge ou CK), PCK et le développement du PCK.

- La première contribution porte sur les CK d'enseignants anglais du primaire en formation initiale et la confiance qu'ils attribuent à ces connaissances. Elle discute aussi les relations entre ces deux éléments et leurs implications pour la pratique, par exemple dans le cas où les enseignants ne développent pas d'enseignement scientifique parce qu'ils n'ont pas confiance dans leurs $\mathrm{CK}$ alors qu'elles sont pertinentes, ou à l'inverse, dans le cas où les enseignants n'hésitent pas à développer des séances de sciences parce qu'ils ont confiance dans leurs CK alors que celles-ci sont déficientes. 
- Le chapitre suivant présente un modèle pour l'acquisition de connaissances professionnelles basé sur les régulations rendues nécessaires par l'apparition d'évènements inattendus, modifiant en séance l'activité planifiée (boucle de rétroaction courte) et ayant des conséquences à plus long terme sur la planification et l'enseignement de la même séance l'année suivante (boucle de rétroaction longue). Ce modèle, fondé sur la théorie de l'activité (Léontiev, 1975), la clinique de l'activité (Clot \& Faita, 2000), et didactique professionnelle (Pastré, 1999) est mis à l'épreuve lors d'une étude longitudinale de l'implémentation d'une démarche d'investigation par deux enseignants de collège français. Les connaissances professionnelles identifiées sont mise en relation avec le modèle du PCK proposé par Magnusson et al. (1999) que les auteurs jugent complémentaire du leur.

- Après avoir discuté le modèle issu du PCK Summit, la contribution suivante propose un modèle mettant en interrelation les PCK, considérées principalement comme des connaissances pratiques, l'action des acteurs et le contexte dans lequel elles se déroulent. Actions et contexte sont décrits dans le cas où les élèves rencontrent des difficultés dans un cours de chimie d'un lycée français, et les PCK sont inférées de cette description, ce qui permet de discuter de leur pertinence au regard de ce que les élèves produisent. Les auteurs discutent la difficulté qu'ils ont à relier les éléments qu'ils infèrent, associés au contrat didactique, aux modèles des PCK, notamment à celui de Magnusson et al. (1999), "that was built more for 'PCK on action' than 'PCK in action'” (p. 58). Ils soulignent en conséquence l'absence de modèle d'enseignement-apprentissage explicite fondant la catégorisation des PCK, ce qui d'ailleurs pourrait aussi expliquer la multiplicité des modèles proposés au cours du temps.

- Le dernier texte de cette première section analyse les PCK des professeurs à la lumière de la théorie de l'action conjointe en didactique (Sensevy, 2011). L'auteur aboutit à une formalisation originale des PCK impliquant les concepts de cette théorie. La formalisation met en effet en relation à propos d'un problème lié à un objet de savoir particulier, le jeu épistémique de référence, la dialectique « contrat - milieu » (c'est-à-dire entre connaissance du système de stratégies disponibles pour les acteurs et conception d'un milieu donnant une structure symbolique au problème »), la dialectique " expression - réticence » associée à la part variable des significations cachées ou révélées par le professeur lors de l'avancée des savoirs, et le système de stratégies contrefactuelles que le professeur est capable d'imaginer en lien avec l'ensemble des éléments précédents.

5 Finalement, ce sont donc quatre textes aux contenus très différents, au demeurant tous intéressants lorsqu'ils sont pris indépendamment, mais qui de mon point de vue peinent à être rassemblés sous un intitulé unique, relatif à l'importance du contenu enseigné au regard de l'efficacité de l'enseignement. Par contre, si on exclue le premier, on peut lire l'intérêt que les catégorisations des connaissances professionnelles trouveraient à être supportées explicitement par des théories liées à l'enseignement et l'apprentissage.

6 La section 2 "Balancing general and specific pedagogical knowledge: role of collaborative settings" comporte à l'instar de la précédente, quatre chapitres et est centrée sur les liens entre les PCK et les PK, ainsi que sur la nature de ces dernières.

-L'activité comparée de deux professeurs de chimie, l'un expérimenté, l'autre novice, implémentant chacun dans un lycée français dans le cadre d'une courte séance de formation continue une séance discutée collectivement, constitue le support empirique de la première étude présentée. L'analyse de l'activité des deux enseignants est fondée à la fois sur la double approche ergonomique et didactique (Robert, 2008), sur le modèle de Magnusson et 
al. (1999), et sur un modèle formalisant les PK. En même temps qu'elle donne des critères pour les différencier, elle montre l'importance à la fois des PK, en partie absentes chez le professeur novice, et des PCK, sur le déroulement de la séance implémentée.

- Le chapitre suivant fonde le développement des connaissances professionnelles sur la théorie de l'activité (Engeström, 2001) et examine en les distinguant les PK et les PCK de 18 professeurs (experts engagés dans la formation initiale, expérimentés, et novices) menant une séance mettant en jeu l'argumentation dans une démarche d'investigation. De manière un peu inattendue, l'activité des novices tend à montrer un répertoire de PCK plus développé que celui de leurs collègues, en lien avec leur récente formation, alors que le répertoire des experts-formateurs relatif aux PK dont la séance analysée fait montre est beaucoup plus important que celui lié aux PCK. La conclusion insiste sur l'importance du maintien d'un équilibre entre les deux formes de connaissances dans la formation initiale et continue des enseignants.

- Le troisième chapitre de cette section est centré sur le développement de PCK relatives à l'enseignement par "inquiry" dans un contexte collaboratif chez cinq éducateurs scientifiques de deux jardins botaniques espagnols. Le développement est pensé en référence à la théorie de l'activité (Engeström, 2001) et à un point de vue complémentaire formalisant le transfert des connaissances tacites des experts vers les novices. Selon les résultats présentés, le projet a permis un développement professionnel significatif au sein de la communauté concernée.

- La dernière contribution de cette partie de l'ouvrage défend et illustre à partir du cas de trois enseignants suédois, l'idée que les PCK et les PK peuvent être développées à partir d'une réflexion critique et collaborative sur les séances qu'ils mènent en classe, dans le cadre d'une "Learning stuydy". Il s'agit d'un processus collégial et cyclique de Design-Based Research dans lequel les professeurs réfléchissent avec un chercheur sur les conditions nécessaires pour apprendre un contenu spécifique, mettent en œuvre la séance conçue, analysent la pratique correspondante, en tirent des conclusions et recommencent en affinant la mise en œuvre.

7 En continuité avec la section précédente, la section 2 confirme donc l'intérêt d'articuler toute formalisation des connaissances professionnelles à un cadre théorique permettant de plus de penser leur évolution, notamment dans un contexte de projet collaboratif. La théorie de l'activité (Engeström, 2001) remplit cette condition, en étendant le point de vue vygotskien d'un apprentisage socio-culturel et en le combinant à celui porté par Lave et Wenger (1991) d'un apprentissage situé dans une communauté de pratiques.

8 La section 3 propose deux contributions alternatives.

- La première est liée à une importante étude empirique concernant une enseignante israélienne experte en enseignement présentiel, chargée de mettre en place une classe virtuelle en physique de niveau lycée. Dans l'étude, la capacité de la professeure à utiliser des outils technologiques nouveaux pour elle est regardée comme une sensibilité à des affordances de ces outils caractérisant une partie de ses connaissances professionnelles. En examinant le corpus à la lumière de ce point de vue, complété par des théories formalisant l'appropriation et la structuration des connaissances individuelles, l'auteure produit une modélisation originale dans laquelle elle relie, le système de connaissances existant chez le professeur, la reconnaissance d'affordances dans les ressources et les outils nouveaux à sa disposition, l'appropriation et l'intégration dans le répertoire d'actions éducatives de ces ressources et outils, leur mise en œuvre dans la classe et la modification en conséquence du 
système de connaissances de l'enseignant. Ce modèle est alors comparé au modèle du PCK summit avec lequel il partage certains éléments, alors que d'autres lui sont totalement étrangers, voire incompatibles avec lui.

- La seconde contribution alternative explore avec un point de vue cognitif, les connaissances professionnelles des enseignants. Pour les auteurs, des connaissances qu'ils qualifient de fondamentales et qui sont associées à de nombreuses capacités humaines mobilisées dans des contextes communicationnels et sociaux, peuvent être aussi mobilisées dans l'enseignement de manière automatique ou presque, au moins tant que la charge cognitive à laquelle l'enseignant doit faire face n'est pas trop élevée. Ils défendent aussi l'idée que la distinction qu'ils opèrent entre connaissances primaires, acquises par adaptation à partir de l'expérience et connaissances secondaires, acquises par éducation peut être appliquée pour distinguer les PK, associées à la gestion des interactions sociales entre professeur et élèves, des PCK qui concerneraient la conception de séances et les stratégies purement didactiques.

Enfin, une conclusion riche clôture l'ouvrage, articulée autour de l'exploitation de deux points de vue complémentaires, en référence aux contributions précédentes. D'une part, l'enseignement est une activité professionnelle relevant des activités humaines, et comme les autres, elle contribue à la formation de contextes humains et sociaux, dans lesquels la connaissance est en évolution constante. D'autre part, l'enseignement est une activité spécifique dans la mesure où elle concerne des contenus particuliers à transmettre, des professeurs avec leurs croyances et leurs idéologies, des élèves avec leurs connaissances préalables ; cette activité professionnelle nécessite en conséquence des connaissances particulières mises en œuvre dans un contexte spécifique. A partir de la discussion de chacune des contributions de l'ouvrage, un nouveau modèle des "Science Teachers' Professional Knowledge in Contexts" (TPKinCs) est progressivement construit. Il met en relation (p. 220) professeur et contextes. Le professeur est caractérisé par une base de connaissances professionnelles, propres à tous les membres de la communauté éducative scolaire, une base de connaissances pédagogiques générales, propres à la communauté enseignante, une base de connaissances spécifiques à l'enseignement d'un domaine disciplinaire et par un ensemble de filtres et d'amplificateurs individuels (croyances, orientations, sensibilité aux technologies, etc.) conditionnant l'activité développée en classe. Les contextes quant à eux sont au nombre de trois : 1) le contexte instrumental, incluant les ressources, les outils, et la formation professionnelle; 2) le contexte social, incluant la communauté des professeurs, la division et l'organisation du travail en son sein, les habitudes et répertoire d'actions qui y ont cours ; 3) le contexte de la classe, incluant la pratique de classe et les résultats obtenus par les élèves, en termes de maitrise du contenu, des méthodes scientifiques, etc. Le modèle intègre aussi la notion de connaissance tacite ou explicite et des boucles de régulation, et met en relation cet ensemble d'éléments.

Le modèle TPKinCs élaboré à partir de l'ensemble des contributions proposées et du modèle issu du PCK summit qui a été largement discuté, est donc un point conclusif fort de cet ouvrage, d'autant qu'il est associé à l'idée de développement professionnel et qu'il s'appuie sur un cadre théorique, la théorie de l'activité, sans s'y réduire et en le contextualisant à l'activité enseignante. Moyennant une adaptation partielle relative à la partie disciplinaire du modèle, il pourrait d'ailleurs probablement être exploité audelà des professeurs de sciences. Toutefois, on ne sait pas trop quel caractère (normatif, heuristique, descriptif...) conférer aux bases de connaissances incluses dans le modèle. Par ailleurs, celui-ci résulte de l'association d'éléments empruntés dans les 
contributions successives liées à des points de vue théoriques différents et leur compatibilité épistémologique mériterait d'être interrogée. Il reste maintenant aux chercheurs intéressés à mettre ce modèle à l'épreuve pour avoir une idée de son destin scientifique, un modèle qui est somme toute assez complexe si on veut l'exploiter dans son ensemble, mais qui marque l'insertion des didacticiens français des sciences dans le courant des travaux sur les PCK et plus largement dans celui de la formalisation des connaissances professionnelles des professeurs de sciences, fortement développé au plan international.

11 Un autre point fort de l'ouvrage réside dans les résultats proposés dans la plupart des contributions, dont certains sont parfois inattendus (par exemple connaissances de type PCK interprétées comme des obstacles à la réussite des élèves, quasi-absence des PCK au profit des PK chez des professeurs formateurs) et d'autres ouvrent des pistes pour la formation (développement professionnel significatif associé à des activités collaboratives, souvent itératives, menées dans des groupes d'enseignants, souvent avec des extérieurs, parfois formateurs, parfois chercheurs). Par contre, comme dans de nombreux articles ou ouvrages en lien avec les PCK, les résultats ne permettent pas de documenter largement les connaissances utiles à mettre en œuvre pour enseigner telle ou telle partie des savoirs disciplinaires dans tel ou tel contexte Mais peut-être cette idée contient-elle une certaine utopie, ce qui expliquerait pourquoi depuis que Shulman a introduit l'idée des PCK, les travaux et les controverses ont beaucoup plus porté sur les modèles, sur la structure et les différentes catégories de connaissances professionnelles, que sur les connaissances elles-mêmes.

Enfin, une partie de l'ouvrage met en avant les outils théoriques et méthodologiques développés par les didacticiens francophones pour analyser les pratiques enseignantes, qui contribuent ici à apporter une première réponse à une des questions soulevées au PCK summit sur la «capture » des PCK, notamment celles manifestées dans l'action. Rédigé en anglais, l'ouvrage permet ainsi de faire connaître ces outils à l'étranger, et facilite pour l'avenir d'autres publications internationales des chercheurs francophones, en lien ou non avec les PCK. A ce titre, et au-delà de la richesse des travaux présentés, on ne peut qu'en remercier tous les contributeurs.

\section{BIBLIOGRAPHIE}

Abell S.K. (2007) Research on Science Teacher Knowledge. In S.K. Abell and N.G. Lederman (Eds), Handbook of Research in Science Education (pp. 1105-1149). London : Laurence Erlbaum associates.

Biological Sciences Curriculum Study (2012). PCK Summit . Welcome to The PCK Summit

Dissemination Site, [en ligne]. http://pcksummit.bscs.org/ (page consultée le 04-02-2016)

Clot, Y., \& Faita, D. (2000). Genres et styles en analyse du travail : concepts et méthodes. Travailler, $4,7-42$

Engeström, Y. (2001). Expansive learning at work: Toward an activity theoretical reconceptualization. Journal of Education and Work, 14(1), 133-156. 
Gess-Newsome, J. (1999). Secondary teachers' knowledge and beliefs about subject matter and their impact on instruction. In J. Gess-Newsome, \& N. Lederman (Eds), Examining pedagogical content knowledge: the construct and its implications for Science Education. Bonston (MA) : Kluwer Gess-Newsome, J. (2015). A model of teacher professional knowledge and skill including PCK: Result of the thinking of the PCK summit. In A. Berry, P. Friedrichsen, \& J. Loughran (Eds), Reexamining pedagogical content knowledge in science education (pp. 28-42). New-York (NY): Routledge. Grangeat, M. (2015). Understanding Science Teacher's Professional knowledge Growth. Rotterdam : Sense Publisher

Grossman, P. L (1990). The making of a teacher: Teacher knowledge and teacher education. New-York : Teachers College Press.

Hattie, J. (2003, October). Teachers make a difference: What is the research evidence? Paper presented at the Australian Council for Educational Research Annual Conference on Building Teacher Quality, Melbourne. Accessible le 02-02-2016 à http://klas4a.free.fr/f2ks/ RC2003_Hattie_TeachersMakeADifference_1_.pdf

Léontiev, A. (1975). Activité, conscience, personnalité. Moscou : Editions du progress.

Magnusson, S., Krajcik, J., \& Borko, H. (1999) Nature, Sources, and Development of Pedagogical Content Knowledge for Science teachers. In Gess-Newsome, J., \& Lederman, N.G. (Eds.), Examining Pedagogical Content Knowledge: The Construct and its Implications for Science Education (pp. 95-132). Boston : Kluwer Academic Publishers

Pastré, P. (1999). La conceptualisation dans l'action. Bilan et nouvelles perspectives. Education Permanente, 139, 13-35.

Robert, A. (2008). La double approche didactique et ergonomique pour l'analyse des pratiques d'enseignants de mathématiques. In F. Vandebrouck (Ed.), la classe de Mathématiques : activités des élèves et pratiques des enseignants (pp. 59-68). Toulouse : Octarès.

Sensevy, G. (2011). Le sens du savoir. Bruxelles : De Boeck.

Shulman, L.S. (1987).Knowledge and teaching: Foundation of the new reform. Harvard Educational Review, 57, 1-22.

\section{AUTEURS}

\section{PATRICE VENTURINI}

UMR EFTS, Université de Toulouse Jean Jaurès 